\title{
Kernos
}

Revue internationale et pluridisciplinaire de religion grecque antique

21 | 2008

Varia

\section{Ex decretis prioribus nihil immutamus. Du conservatisme religieux des Romains}

John Scheid

\section{(2) OpenEdition \\ Journals}

Édition électronique

URL : https://journals.openedition.org/kernos/1614

DOI : 10.4000/kernos.1614

ISSN : 2034-7871

Éditeur

Centre international d'étude de la religion grecque antique

Édition imprimée

Date de publication : 1 janvier 2008

Pagination : 185-196

ISSN : 0776-3824

Référence électronique

John Scheid, «Ex decretis prioribus nihil immutamus. Du conservatisme religieux des Romains », Kernos [En ligne], 21 | 2008, mis en ligne le 01 octobre 2011, consulté le 24 août 2022. URL : http:// journals.openedition.org/kernos/1614 ; DOI : https://doi.org/10.4000/kernos.1614 


\section{$E_{X}$ decretis prioribus nihil immutamus. Du conservatisme religieux des Romains}

Dans son livre sur quelques frères arvales ${ }^{1}, \mathrm{R}$. Syme cite la phrase suivante qu'il empruntait à ces prêtres : "[De institut]is prioribus nihil immutamus.» Cette formule qu'il présente comme unique, lui paraît abnormal and superfluous, parce que ce collège sacerdotal serait entièrement marqué par le conformisme et l'inclination - adherence - vers la tradition. De cette manière, il put constater que le collège arvale n'a recruté que des médiocrités insignifiantes, qui montraient peu d'intérêt pour l'innovation et pour l'orgueil.

Ces déductions sont sans doute superficielles et déforment quelque peu les faits. Même si le lecteur moderne apprécie le style incomparable de Sir Ronald, son humour narquois et sa critique spirituelle de toute forme de religion, - et je suis parmi ceux qui aiment ces qualités - je ne puis pas le suivre sur ce point. C'est pourquoi je voudrais profiter de cette occasion pour discuter de cette formule, en me rappelant l'enseignement de J. Rudhardt. Quand, en préparant ce texte, j'ai feuilleté son livre sur les Notions fondamentales de la pensée religieuse et actes constitutifs du culte dans la Grèce classique, j'ai retrouvé les notes marginales que j’y avais portées. Elles m'ont rappelé une fois de plus combien les Grecs, les savants de la religion grecque, m'ont appris, et J. Rudhardt en particulier. J'ai notamment découvert deux traits en marge de sa phrase : « il faut comprendre la religion antique selon ses concepts propres, l'expliquer par la pensée antique elle-même $»^{2}$. C'est ce que J. Rudhardt a fait dans son livre, devenu un classique. Je ne peux donc pas mieux honorer sa mémoire qu'en essayant de démontrer que lorsqu'on essaie de comprendre selon les concepts propres des Romains la formule arvale moquée par R. Syme, elle prend un sens très précis, qui n'a rien à voir avec la médiocrité des arvales ou le côté réactionnaire des Romains. Voyons donc le problème d'un peu plus près.

1 R. Syme, Some Arval Brethren, Oxford, 1980, p. 110 : «Conformity appeals all through, not least to the new senator, and adherence to tradition. On one occasion the Acta produce an abnormal but superfluous declaration: '[de institut]is prioribus nihil immutamus'. » Déjà en 1958, dans Tacitus I, Oxford, 1958, p. 66, Syme avait qualifié les arvales de nonentities: « Nonentities now tend to congregate among the arval Brethren... » Je dois cette référence à M. Christol.

2 J. RUDHARDT, Notions fondamentales de la pensée religieuses et actes constitutifs du culte dans la Grèce classique. Étude préliminaire pour aider à la comprébension de la piété athénienne au IVe siècle, Genève, 1958, p. 7. 
J'ai passé une grande partie de ma vie active dans la confrérie arvale, et je n'y ai constaté aucune des qualités que Sir Ronald a attribuées à ces prêtres. Dans une vie antérieure, quand je pratiquais activement la recherche prosopographique, et que je fichais tous les prêtres connus de la Rome impériale, j’ai pu constater que les reproches que R. Syme adresse aux arvales flaviens peuvent, en fait, être formulés à propos de tous les collèges sacerdotaux, même les plus brillants ${ }^{3}$. Syme considérait comme non-médiocrité des sénateurs qui remplissaient les charges publiques les plus élevées, et qui pour cette raison sont d'ailleurs bien traités par les sources. Tous les autres, dont les sources antiques ne parlent guère, étaient pour lui des nonentities, de médiocres petits sénateurs. Des collègues oxoniens m'ont confié que dans la petite prosopographie des arvales flaviens, R. Syme brossait en fait un portrait de ses collègues d'Oxford. Je suis incapable de le confirmer, mais on peut effectivement sentir derrière ce livret une certaine amertume rétrospective jointe à un jugement sévère des tranquilles et médiocres fellows grandis dans le sérail, qui devaient regarder de très haut le brillant Néozélandais dont ils n'atteignirent jamais la réputation scientifique ni la gloire internationale. Comme l'écrit R. Syme, l'arvale ne voyait aucune raison de critiquer un club qui n'acceptait personne qui fût meilleur que lui-même. Et dans un club de ce genre un R. Syme dérangeait.

Néanmoins, malgré toutes les explications érudites du livre, la définition générale du rang social des frères arvales est excessive, parce que Sir Ronald oublie la fonction des arvales. Étant tous de rang sénatorial, autrement dit, appartenant à ce tiers éminent des sénateurs qui possédaient une dignité sacerdotale, ces douze prêtres célébraient chaque année un culte public, un sacrifice qui appartenait aux obligations religieuses du Peuple romain, c'est-à-dire de l'État romain. Ce rôle dément l'appréciation de Syme; et si l'on considère en outre que l'empereur faisait partie de la confrérie, qu'il en exerçait la présidence quand son tour arrivait, et que l'institution fonctionna jusqu'à l'époque de Dioclétien, il apparaît qu'il est exagéré de parler de médiocrité ennuyeuse et hyperconservatrice. Pour que cette description fût exacte, il faudrait que Syme prouve que les autres collèges sacerdotaux étaient sur ce point différents des arvales. Or il n'envisage même pas cette comparaison et, si on la fait, on découvre qu'il y avait autant de nonentities dans les grands collèges sacerdotaux que chez les arvales.

Mais venons-en à la formule incriminée par Sir Ronald : [de institut]is prioribus nibil immutamus. Cette formule serait unique, écrit-il. Ceci est faux. Sir Ronald a copié la formule dans l'Année épigraphique de 1964, sans feuilleter les autres documents arvales. La formule qui figure sur ce protocole de l'année 109 - à l'époque de Syme on le datait encore de 110 - n'est pas unique; 25 ans plus tard, elle est à nouveau employée par les frères. Mais lisons d'abord la formule

${ }^{3}$ J. SCHEID, Le collège des frères arvales. Étude prosopographique du recrutement (69-304), Rome, 1990, p. 266-312. 
dans son contexte. Nous sommes le 19 mai 109, à 8 km à l'ouest de Rome, au bois sacré de Dea Dia. Cette déesse s'occupe de la bonne lumière durant la période de la maturation définitive des céréales. En ce jour, le collège offrait à Dea Dia le sacrifice annuel d'une agnelle grasse, qui occupait toute la journée. L'après-midi, après le sacrifice qui débutait à midi, les arvales consommaient un banquet sacrificiel, un épisode rituel qui a déjà bénéficié jadis des sarcasmes de Th. Mommsen 4 , à qui il rappelait sans doute les banquets des monsignori de Rome. Après ce banquet, les prêtres se rendaient dans le cirque voisin et lançaient le signal de départ aux quadriges. Les pauses entre les courses du cirque ont depuis toujours offert aux Romains l'occasion de présenter des doléances au maître du lieu ou au président des jeux. C'est ce qui se passa en 109 au cirque près du bois sacré de Dea Dia, vers cinq heures de l'après-midi. L'état du protocole ne permet pas de savoir qui présente la doléance ni pour quelle raison. Seule la formule citée par Syme est partiellement conservée. Lisons ${ }^{5}$ :

Après le banquet, [le vice-président Caecilius Simplex portant le ricinium, des sandales et une couronne de roses tressée, après avoir fait écarter les assistants,] monta au-dessus des barrières et [lança] le signal aux quadriges [et aux cavaliersvoltigeurs. Sous la présidence de --- et de Trebicius] Decianus, il [décora] les vainqueurs de palmes et de couronnes [d'argent. <Interrogés à propos de $>---$,] Catius Fronto, Metilius Nepos, Trebicius Decianus, $---<$ décidèrent : «À propos de $>---$, nous ne changeons rien [des décrets] antérieurs. »

Le deuxième témoignage est un peu plus complet. Le contexte est le même. Lors des jeux du cirque concluant le sacrifice à Dea Dia, le 29 mai 134, les esclaves publics se présentèrent devant les arvales pour en exiger une décision. Le début du texte est presque incompréhensible, et contient d'ailleurs le joli barbarisme etulitum qui doit appartenir au verbe efferre, et serait donc elatum. Efferre, comme processus inverse de inferre, deferre, "déposer, enregistrer aux archives ", signifierait donc que quelque chose a été extrait des archives. Nous pouvons comprendre dans la suite qu'il s'agissait d'un décret (decretum « etulitum ») des arvales. Lisons ${ }^{6}$ :

${ }^{4}$ Th. Mommsen, Über die römischen Ackerbrüder. Vortrag gehalten in der Singakademie zu Berlin, 22 Januar 1870, republ. dans Th. MommSEN, Reden und Aufsätze, Berlin, 1905, p. 286-288.

${ }^{5} \mathrm{~J}$. SCHEID, Commentarii fratrum arvalium qui supersunt. Les copies épigraphiques des protocoles annuels de la confrérie arvale (21 av.-304 ap. J.-C.), Rome, 1998, p. 190 sq., nº 65 (109 ap. J.-C.) : Post ep[ulas riciniatus soliatus corona pactili rosacia Caecilius Simplex promagister sum-] / [moto super ca]rceres ascendit et signum quadrigis [et desultoribus misit praesidentibus - - - / [ - - Trebici]o Decianó uictores palmis et coronis [argenteis honorauit. - - ] / [- - , Ti. C] alt thus Fronto, Metilius Nepos, Trebicius [Decianus, - ----$.$] / [- - ex decret ]$ is prioribus nibil immutamus.

${ }^{6}$ SCHEID, o.c. (n. 5), p. $223, \mathrm{n}^{\circ} 75$ (134 ap. J.-C.) : I sdem co(n)s(ulibus) (ante diem quartum) k(alendas) Iun(ias) / [ - -, Iul(ius) Alexander] Iulianus, Antonius Albus, Valerius Iunianus / [ - publicis s]uis postulantibus, ut ex sententiis fratr(um) aru(alium) / [- - ?i]n portionibus aput ipsos etulitum (!) Eutychen / [ - - perl]ectis codicibus, quibus sententiae priorum / [fratr(um) aru(alium) relatae sunt, collegium decreu] it ou collegio placu] it: «Ex decretis prioribus nibil / [immutamus, - - port]io circi concessum a collegio nostro public(is ?). » 
Sous les mêmes consuls, le quatrième jour avant les calendes de juin, [- - -, Iulius Alexander] Iulianus, Antonius Albus, Valerius Iunianus,,$---<$ furent interpellés ?> par] leurs [esclaves publics ?] demandant que (le décret ?) extrait des décisions des frères arvales $[---]$ dans les portions auprès d'eux-mêmes, Eutyches $[---$, après avoir lu les registres dans lesquels [sont consignées] les décisions des [frères arvales] antérieurs, [le collège décréta: «Nous ne changeons] rien des décrets précédents. [- - ] la portion du cirque (- - (quelque chose) ] est attribué par notre collège aux esclaves publics [-- ]. »

Je ne discuterai pas du sens de portio ni de ce que les esclaves demandent et reçoivent ou non. Ce n'est pas la portio elle-même, car ce qui est accordé ou non est un neutre (concessum). On a pensé à un terrain situé près du cirque, ou à des places au cirque. Quoi qu'il en soit, le décret des arvales comprend la formule signalée par R. Syme : de ce qui a été décidé précédemment, on ne change rien. Cette fois-ci l'état de la pierre révèle que ce dont rien n'est changé ce sont les décisions des arvales, les décrets exprimant les sententiae, les décisions des arvales précédents, et c'est ainsi qu'il convient aussi de restituer la lacune dans la pierre de 109. Ce passage nous apprend aussi que les décrets des arvales étaient enregistrés et consultés sur les codices, les tablettes enduites de cire, et non sur les copies épigraphiques. Vraisemblablement ces tablettes portaient le sceau ou la subscriptio du magister annuel qui attestaient leur authenticité, alors que les inscriptions fixées sur une paroi au bois sacré ne possédaient pas cette qualité. Les protocoles sur lesquels figuraient les décrets en question étaient donc déposés dans un bâtiment du bois sacré.

$\mathrm{R}$. Syme a-t-il raison de considérer la formule ex sententiis prioribus nibil immutamus comme un signe du conservatisme stérile et médiocre des arvales?

La réponse sera encore négative. D’abord parce que les arvales étaient loin d'être les seuls à raisonner de cette manière. Il s'agit d'une attitude bien plus générale, comme le démontre le choix de témoignages suivants.

Dans ses Lois, Cicéron ${ }^{7}$ écrit que les sages prescriptions cultuelles que Numa avait faites quant à la gestion du calendrier annuel furent oubliées en raison de la négligence des pontifes postérieurs (posteriorum pontificum). Il pose ensuite la règle suivante: Parmi les coutumes de pontifes et des haruspices on ne devra rien changer des règles qui décident quelle victime animale doit être offerte à quel dieu, auquel des victimes adultes, auquel des victimes à la mamelle, auquel des animaux mâles, auquel des femelles. Illud de institutis pontificum et haruspicum non mutandum est, etc. Cicéron emploie donc pratiquement la même formule que les arvales. Cent-trente années plus tard, en 90, l'empereur Domitien écrivit une

\footnotetext{
${ }^{7}$ Cicéron, Lois II, 22 : Ex patriis ritibus optuma colunto; II, 29 : Quod <ad> tempus ut sacrificiorum libamenta seruentur fetusque pecorum quae dicta in lege sunt, diligenter habenda ratio intercalandi est, quod institutum perite a Numa, posteriorum pontificum neglegentia dissolutum est. Iam illud ex institutis pontificum et haruspicum non mutandum est, quibus hostiis immolandum quoque deo, cui maioribus, cui lactentibus, cui maribus, cui feminis.
} 
lettre au Delphiens ${ }^{8}$. Le conseil des Amphictyons avait apparemment décidé, contre la volonté des Delphiens, de changer la date des fêtes pythiques. Les Delphiens portèrent plainte auprès de l'empereur, qui avait réparé et restauré en 84 le temple d'Apollon. L'Empereur Domitien répondit qu'il était conforme à la justice et à la piété naturelles que la date des Pythia soit conservée en conformité avec les coutumes des Amphictyons, et que l'on ne bouleverse aucune partie des coutumes antiques. En latin, je traduirai le principe qu'il rappelle : de institutis prioribus nibil immutandum ...

Le même principe que celui des arvales est affirmé par Cicéron dans les Lois, puis par Domitien : il ne faut rien changer aux coutumes et décisions rituelles. Chez les arvales, nous ne savons pas exactement de quoi il s'agit, dans un cas c'est apparemment un privilège qui est en cause, dans l'autre nous ignorons l'enjeu. En tout cas, chez Cicéron et Domitien, le principe se rapporte clairement à des rites. Ceci nous permet donc de constater qu'en matière de religion et de traditions relatives à des cultes et des temples, les Romains observaient le principe général de ne rien changer à la tradition précédente. Il est intéressant de noter que Domitien applique la règle au sanctuaire de Delphes, en traduisant même la formule que nous étudions. Dans son livre sur l'Amphictyonie de Delphes, P. Sánchez ${ }^{9}$ constate que Domitien emploie ce principe parce que la loi ou coutume ne contrevenait pas aux intérêts de Rome. J'ajouterai que pour trancher ce conflit grec, l'Empereur se réfère à un principe romain. À moins que le principe ne soit universel ?

Serait-ce donc un principe partagé par les Grecs et les Romains ? Plusieurs autres témoignages paraissent le prouver. Dans le Contre Nicomachos, attribué à Lysias $^{10}$, ce dernier ridiculise Nicomaque qui avait eu la charge de transcrire les nomoi pâtrioi en 410/9, et il avait rempli cette tâche pendant six ans. Je passe sur l'accusation dont il fut l'objet en 399, pour m'intéresser uniquement à un argument employé par Lysias. Celui-ci se moque de Nicomachos, parce que celui-ci l'accuse «d'impiété pour avoir dit qu'il faut accomplir les sacrifices prescrits par les tables et les stèles conformément aux ordonnances ». Il constate qu'ainsi c'est la cité même que Nicomachos incrimine : «car ce sont (les) décrets qui ont ainsi décidé » ${ }^{11}$.

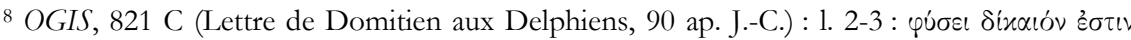

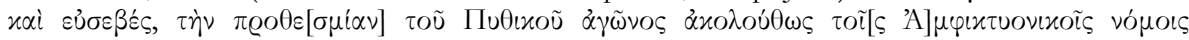

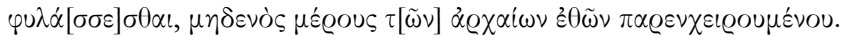

${ }^{9}$ P. SÁNCHEZ, L'Amphictyonie des Pyles et de Delphes. Recherches sur son rôle historique des origines an IIe siècle de notre ère, Stuttgart, 2001 (Historia Einzelschriften, 148), p. 450.

${ }^{10}$ Lysias, C. Nic. (30).

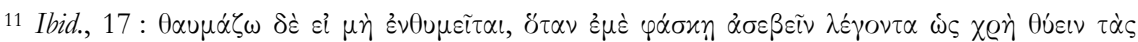

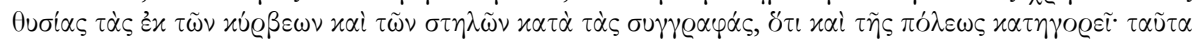

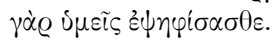


De même, Isocrate proclame dans l'Aréopagite ${ }^{12}$, que, « en ce qui concerne les dieux (c'est par là qu'il est juste de commencer), ce n'est pas sans régularité ni ordre qu'ils (c'est-à-dire les ancêtres) les servaient ou célébraient leurs mystères... Ils ne veillaient qu'à une chose: c'était à ne rien supprimer des traditions ancestrales et à ne rien ajouter qui sortît des usages reçus. Ce n'est pas dans le luxe qu'ils faisaient résider la piété, mais dans le fait de ne toucher à rien

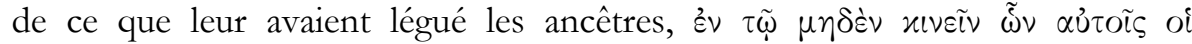
$\pi \varrho o ́ \gamma o v o \iota ~ \pi \alpha \varrho \varepsilon ́ \delta o \sigma \alpha \nu »$.

Mais la liste ne s'arrête pas là. Dans le Dialogue sur les oracles de la Pythie, Plutarque ${ }^{13}$ écrit, à peu près à l'époque du premier décret des arvales, que « l'on ne doit pas accuser le dieu ou l'oracle, sa prudence et sa divinité, mais qu'on doit chercher la solution des contradictions apparentes et non abandonner la piété et la foi de nos pères ». Et quand le thiase athénien des Iobacchoi ${ }^{14}$ fut refondé en 178, il se contente de faire acclamer les dogmata, en latin les decreta, des prêtres précédents, et à les faire écrire sur une stèle. Même l'empereur Julien écrit encore dans sa lettre au prêtre Theodore, à qui il explique les grandes lignes de sa réforme de l'autorité sacerdotale : «Je fuis dans tous les domaines le changement, je peux l'affirmer, et avant tout dans ce qui concerne les dieux. Je crois que l'on doit se tenir aux lois que nos pères avaient dès le début, et qui semblent être un don des dieux $»^{15}$.

Par conséquent, la formule qui affirmait qu'il n'y avait rien à changer à une vieille tradition n'est pas seulement un principe arvale, mais se retrouve sous le calame de l'empereur Julien, des thiasotes d'Athènes ou des philosophes et orateurs d'Athènes et de Rome, toujours en rapport avec les rites et les prescriptions religieuses. C'est en fait un des principes fondamentaux des religions du monde classique. Dans le domaine religieux, on ne doit pas toucher aux règles cultuelles ni les changer. Les prescriptions reprises aux prédécesseurs doivent demeurer telles quelles. Elles doivent durer éternellement, comme les

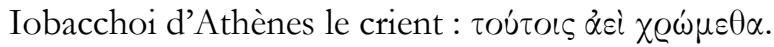

Et cette maxime n'était pas seulement religieuse, mais elle concernait aussi les lois en général. Comme J.-M. Bertrand l'a montré dans son De l'écriture à l'oralitét ${ }^{16}$ c'est le principe même de la législation et de la politique, d'après

12 Isocrate, Aréop. (7), 30.

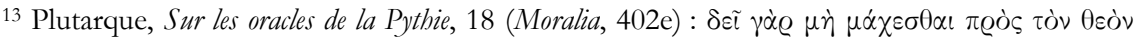

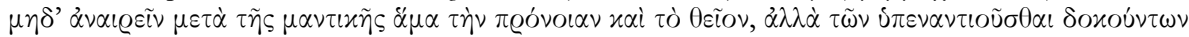

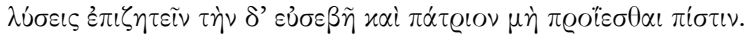

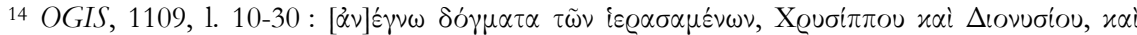

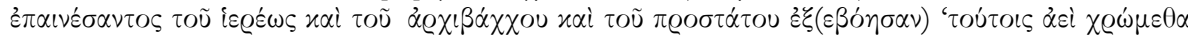

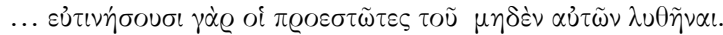

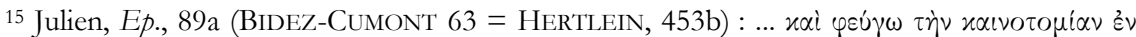

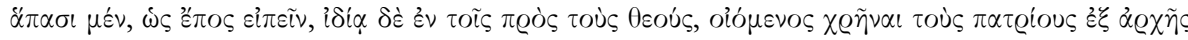

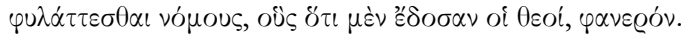

${ }^{16}$ J.-M. BerTRAnd, De l'écriture à l'oralité. Lectures des Lois de Platon, Paris, 1999. 
Platon. «Le recours au passé lointain, écrit-il, devait permettre de montrer que le groupe politique n'avait jamais rompu avec ses origines et perpétuait sans avoir rien oublié tout ce qui avait fait la trame d'un passé dont on souhaite montrer qu'il n'avait rien détruit. »Et même si la pratique démentait ce fait, on faisait l'éloge de l'immobilité de la loi.

Sur le plan religieux, ce principe a été longtemps mal compris. Signifie-t-il qu'au fond les religions de l'antiquité gréco-romaine étaient ultraconservatrices, qu'il s'agisse de Rome ou d'Athènes, depuis que l'on sait que les religions de Grèce n'étaient pas moins ritualistes que celles des Romains ? Est-ce que ce conservatisme de la norme, et notamment de la norme écrite est l'indice d'une sclérose du sentiment religieux ? Est-il un symptôme d'une religion sacerdotale ou d'État, qui réprimait toute émotion et se transformait en une sorte de piété d'épicier, pour employer la formule de Th. Mommsen. C'est ainsi que l'on a effectivement considéré les religions de l'Antiquité, en tout cas celles de la Rome antique. Celle-ci consistait dans l'observation la plus précise de la règle cultuelle, et n'exigeait ni foi, ni sentiment ou relation personnelles avec Dieu. Dans le système construit à l'époque romantique, tel qu'il est par exemple présenté dans l'œuvre de Hegel ${ }^{17}$, et qui influença certainement Th. Mommsen, Fr. Cumont et leurs successeurs, la relation immédiate de l'homme avec Dieu était interrompue, et une évolution dialectique conduisait des excès des religions asiatiques fondées sur le sentiment, par la relation naïve et fraîche de la Grèce avec Dieu et la nature, à la piété profonde mais vide des Romains. Rome put ainsi recueillir la religion asiatique sous la forme immortelle de la culture grecque et donner finalement naissance au christianisme, c'est-à-dire à une véritable religion.

Si, à l'instar de R. Syme, on est surpris par la formule des frères arvales, on oppose de la même manière que les romantiques deux types de piété et de religion, dont l'une représente l'avenir et le salut, l'autre le passé et la décadence. Une religion fondée sur le sentiment est une religion digne de ce nom, une religion du rite attire le sarcasme et l'ironie. Or encore de nos jours existent beaucoup de religions qui sont fondées sur l'observance de règles rituelles, indépendamment d'une révélation et de livres saints. Prenons uniquement le cas du judaïsme, qui était régulièrement rapproché de la religion romaine, par exemple par Fr. Cumont ${ }^{18}$, en raison de la prééminence de l'obligation rituelle.

17 W.F. HEgEL, Philosophie der Weltgeschichte. II, 3. Die griechische und römische Welt, Edit. Lasson, Bd. IX, Leipzig, 1923, p. 681; id., Vorlesungen über die Philosophie der Religion, Edit. Lasson, Bd. XIII, Leipzig, 1927, p. 214; Th. Mommsen, Römische Geschichte, Berlin, 1854; Fr. Cumont, Les religions orientales dans le paganisme romain, Paris, 1905, 1929.

18 Cumont, o.c. 1929 , p. 25 (citant Réville). 
La raison de la surprise de Syme réside donc dans son incompréhension d'une religion ritualiste. Comment prendre au sérieux une religion qui consiste seulement en rites? ou pour formuler autrement la question : comment le principe énoncé par les arvales et les autres autorités citées peut-il être compris et expliqué ? Car cette formule exprime les fondements de la religiosité grecque et romaine. Je pense que ni les Grecs ni les Romains ne s'infligeaient ces règles et cette conception du religieux par masochisme.

C'est à la fin du XIXe siècle que l'histoire a commencé de rendre justice à la religiosité du monde antique. À Bonn, $\mathrm{H}$. Usener ${ }^{19}$, par exemple, a peu à peu découvert la valeur du culte, qui le rapprochait davantage de la formation des concepts religieux que les spéculations philosophiques et lyriques. Les Götternamen de 1896 étudiaient les noms sous lesquels les dieux étaient invoqués et compris. Autrement dit, ce livre exploitait les témoignages cultuels pour étudier la religiosité des anciens. U. von Wilamowitz-Moellendorf, le prince berlinois de la philologie, avait une conception diamétralement opposée de la religion. Il ne pouvait accepter, écrivit-il à Usener, cette façon d'étudier la piété des anciens, autrement dit par l'étude du culte ${ }^{20}$. Car, poursuivait-il, «Gefübl ist alles, Name ist Schall und Rauch», tout est dans le sentiment, le nom, c'est-à-dire l'invocation cultuelle, c'est seulement du bruit et de la fumée. Et quand Georg Wissowa publia en 1902 son grand ouvrage sur Religion et culte des Romains, il dut se défendre contre ceux qui comme Wilamowitz lui reprochaient de ne pas avoir traité de religion dans son livre. Donc uniquement de rites, d'obligations et d'instructions sacerdotales. Il est amusant de noter qu'il m'est arrivé de bénéficier des mêmes remarques. Wissowa avait découvert le culte au début des années 1890, donc à la même époque à peu près que Usener. C'est sous l'influence involontaire du droit public romain de Th. Mommsen et par sa collaboration à la réédition du calendrier romain dans le tome I du CIL mommsénien, qu'il découvrit l'importance des sources cultuelles. Comme conséquence, il ajouta à la première partie de son manuel, qui est de nature historique et plutôt décevante pour le chercheur d'aujourd'hui, une deuxième partie, qui était vouée aux institutions cultuelles. D'où le titre étrange du livre, «Religion et culte des Romains ». Dans la préface à la $2^{\mathrm{e}}$ édition, Wissowa répondit à ses détracteurs qui lui reprochaient de ne parler que de rites et de règles, que c'était bien cela que les Romains appelaient religion ${ }^{21}$.

Pour comprendre ce principe fondamental de la religion des Anciens, il faut tenter de comprendre le sens de ce ritualisme, et se demander notamment, pourquoi les Anciens préféraient ne jamais rien changer aux traditions cultuelles.

${ }^{19}$ H. UsENER, Götternamen. Versuch einer Lebre der religiösen Begriffsbildung, Frankfurt, 1896.

${ }^{20}$ U. von Wilamowitz-MoelLENDORFF, Brief an Usener vom 7.12.1895, reproduit chez H.J. METTE, Nekrolog einer Epoche..., Lustrum 22 (1979/80), p. 79-81.

${ }^{21}$ G. Wissowa, Religion und Kultus der Römer, Munich, $1912^{2}$, p. VIII. 
La tradition romaine illustre deux manières différentes de réfléchir sur les rites. D'un côté, c'est la règle rituelle qui est présentée comme incontournable et comme seule importante, de l'autre, les rites sont expliqués de mille manières. Le premier type de discours sur les rites s'appelait religion et correspondait à la pietas, la seconde se rapporte à la culture, au savoir sur le monde et sur les choses.

Dans la tradition canonique, les rites n'avaient pas d'autre sens que celui de l'obligation : tel sacrifice doit être offert tel jour, en tel endroit, par tel prêtre ou magistrat. La manière de célébrer le sacrifice était généralement régie par une tradition orale. De temps à autre, des experts religieux devaient intervenir pour résoudre les problèmes qui pouvaient se poser. Expert religieux signifie à Rome magistrats supérieurs, prêtres et sénateurs. Ces autorités discutaient alors des problèmes qui se posaient et consultaient le collège de prêtres compétent. Avec le décret de celui-ci, ils prenaient éventuellement un sénatus-consulte que les magistrats faisaient ensuite appliquer. Dans d'autres cas, plus simples, c'était le magistrat qui consultait directement les prêtres. Le système rituel romain consistait dans cette combinaison de la tradition orale avec la jurisprudence sacrée notée dans les protocoles des collèges sacerdotaux ou des séances du sénat. Or cette tradition ne contient aucune affirmation sur le sens des rites.

Ce n'est pas une spécialité romaine. Il en va de même dans les autres religions fondées sur le rite. Certains collègues comme l'indianiste Fritz Staal en ont déduit que les rites n'avaient en fait pas de sens, et qu'ils se réduisaient à une obligation. Je n'irai pas aussi loin. Les rites ont un sens, mais il ne dépasse pas la partition rituelle, et ne l'emporte jamais sur les autres explications données. Les formules de prière récitées pendant l'activité rituelle le prouvent. Ainsi, dans les prescriptions de Caton l'Ancien"22, sur les sacrifices privés de son domaine, les prières s'adressent à Jupiter dapalis, Jupiter du riche repas, Jupiter que l'on priait d'accepter ce riche repas, daps. Les protocoles des frères arvales trahissent eux aussi le fait que, pour les prêtres, un sacrifice était un repas servi aux divinités. Car, pendant le sacrifice à Dea Dia, il y avait une séquence que le secrétaire de la confrérie décrit de la manière suivante : "ils jetèrent son repas à la Mère des Lares ", Matri Larum cenam iactauerunt ${ }^{3}$. Je n'entre pas dans ce rite d'offrande étrange, qui aurait certainement stimulé l'humour de Sir Ronald. Ce qui nous intéresse dans cette formulation, c'est que les prêtres, et sans doute leur prière d'offrande, ou au moins leur secrétaire dans son compte rendu, décrivaient ce rite comme un repas symbolique qui réunissait la déesse et les humains. Ceci prouve que le rite possédait un sens pour les célébrants, un sens littéral qui permettait de le répéter.

\footnotetext{
22 Caton, De agricultura, 132 : Iupiter dapalis, quod tibi ... eius rei ergo macte hac illace dape pollucenda esto.

${ }^{23}$ SCHEID, o.c. (n. 5), p. 334, nº 114 (240 ap. J.-C.), col. II, ligne 24.
} 
Et si l'on y regarde d'un peu plus près encore, on constate que les rites possédaient même pour les Romains un sens plus profond. Au cours de l'acte sacrificiel, par exemple, les célébrants saluaient et vénéraient les dieux en tant qu'immortels et puissants, et donnaient à comprendre par les actes rituels que les dieux occupaient la place suprême dans la hiérarchie du vivant. On pourrait en conclure que le rite sacrificiel contient un énoncé sur le système des choses. Mais cet énoncé n'est pas explicite. Le culte romain ne comprenait ni sermon ni lecture, il consistait uniquement en actes et en prières. Quelques indices, tel le résumé du secrétaire des arvales ou les formules de prière attestent, toutefois, que le sens immédiat et littéral des rites n’était pas inconnu des Romains. Quand les experts religieux discutaient de problèmes rituels, c'était par rapport au sens littéral des cérémonies qu'ils examinaient la difficulté qui était à résoudre. Ainsi devaient-ils, par exemple, tenir compte de la nécessité d'énoncer la suprématie et l'immortalité des dieux. Il reste néanmoins étrange pour nous que toutes ces réflexions et interprétations soient demeurées implicites, et qu'elles n'aient nullement occupé une place prépondérante parmi celles qui étaient attribuées aux rites. Elles n’étaient pas nécessaires religieusement. Seul le devoir religieux était nécessaire, et tout ce qui permettait le déroulement correct du service religieux appartenait en fait à la structure même du rite.

À l'extérieur de la pratique religieuse, les rites comme toutes les coutumes et conduites des Romains étaient interprétés de multiples manières, souvent contradictoires. Cette explication polymorphe des coutumes par le mythe, l'étymologie ou la philosophie est bien connue. Comme il n'existait pas de révélation à Rome ou en Grèce, la voie de la vérité était largement ouverte, ou plutôt la voie des vérités, car celles-ci étaient variées, suivant l'objectif ou le contexte de l'explication ${ }^{24}$. La spéculation prenait son origine dans le rite, mais l'interprétation se référait au monde extérieur en fonction des règles propres au genre d'explication utilisé. Il est intéressant de noter que la spéculation part du contexte rituel, même la spéculation philosophique. Depuis Platon, c'est une vieille habitude de débattre pendant les jours de fête religieux et dans des lieux de culte, comme si les rites suscitaient la spéculation. Les instruments rituels ou les lieux de culte eux-mêmes étaient souvent richement décorés de scènes mythiques de tout genre, qui contenaient une spéculation sur le processus rituel ou s'appuyaient sur lui. Mais ils ne donnaient pas une explication du rituel.

Que pouvaient ressentir ceux qui assistaient au culte? Une bonne partie de la communauté ou des célébrants ne devait rien ressentir du tout, comme dans toute religion. Ou bien ils observaient seulement la chose la plus importante, la seule qui fût incontournable, autrement dit, ils contrôlaient que la cérémonie fût célébrée correctement Tout le reste était une affaire privée, chacun pouvait réfléchir sur ce qu'il voyait, et penser la réalité du monde à partir de ces gestes,

${ }^{24}$ Cf. Symm., Relat. III, 10 : Quid interest, qua quisque prudentia uerum requirat? Uno itinere non potest perueniri ad tam grande secretum. 
de toutes les façons possibles selon leur culture respective. Mais tout ceci n'était ni nécessaire au culte, ni prescrit. Comme l'écrit Ch. Malamoud, "les rites deviennent intelligibles quand on a compris qu'ils sont ce qui rend le monde intelligible $»^{25}$.

Mais revenons à la formule des frères arvales. Vous pouvez comprendre maintenant qu'elle n'était ni révélatrice ni surprenante. D'après ce que j'ai dit, il n'y avait aucune raison pour changer un rite ou une règle religieuse dans la Rome antique ou dans le monde antique. Un rite ne se réfère pas au monde extérieur, mais il est le point de départ de toute spéculation sur la réalité extérieure. Les rites sont au contraire l'expression de la relation avec les ancêtres et avec les dieux des ancêtres. Dans le présent, ils expriment le consensus de la communauté. À Rome, le rite est ce qui unit tous les citoyens, ceux du présent et ceux du passé. Il est facile de comprendre la relation entre les règlements religieux, les lois sacrées, les coutumes rituelles et les lois tout court, dont les Anciens réclamaient la pérennité immobile.

Mais alors, me dira-t-on, pourquoi y a-t-il des restaurations religieuses? Sont-elles la conséquence de l'oubli des rites? Ce paradoxe permettra de préciser mes réflexions. Les Romains inventaient effectivement de nouveaux rites. Mais ce qui est en cause, ce n'est pas à proprement parler l'invention de nouveaux rites. Ce n'était pas ainsi qu'on présentait cette activité; on les qualifiait plutôt comme la restauration de rites anciens qu'on avait oubliés ou de devoirs qu’on aurait négligés par le passé.

Deux exemples. En 204, les Romains transférèrent à Rome la Magna mater de Pergame et de Pessinonte. Cette déesse et son culte, célèbre en raison de l'autocastration des Galles, étaient fort éloignés des traditions romaines. Pourtant l'intégration de ce culte dans le culte romain se fit sans problème. Les rites sauvages des Galles demeuraient limités à l'aire du temple de Cybèle, alors qu'une série de rites étaient transférés du système rituel général des Romains vers la fête annuelle de la déesse. Donc il s'agissait, d'après cette mise en scène, soutenue par l'oracle sibyllin, de la restauration de très vieilles traditions plutôt que d'une importation, d'une invention. Protectrice des Troyens et d'Énée, la Magna mater retournait chez ses descendants.

Le paradoxe provient de ce que, pour les Romains, faire du nouveau n'avait pas de sens. Continuer une tradition était, au contraire, l'expression de la sagesse. Donc, pour innover, il convenait de trouver, comme Auguste, la vieille tradition qui était négligée et qui permettait de construire un nouveau rite. Il fallait avant tout prouver que la tradition avait été oubliée, et qu'elle était restaurée. Et les éventuelles adaptations de ces vieux rites, souvent incompatibles avec la vie sociale de l'époque, dérivaient de ces principes vénérables. Même le culte impérial, qui était une invention indéniable, s'inspirait

${ }^{25}$ Ch. Malamoud, «Présentation », ASSR 85 (1994), p. 6. 
de traditions religieuses bien plus anciennes, comme la vénération du Génie, ou la construction de figures divines qui appartenaient depuis bien longtemps à l'entourage des dieux et qui étaient comme des épiphanies de ses vertus : Fortuna redux, Victoria Aug., Clementia Aug. Rien de révolutionnaire donc dans cette révolution : seule la combinaison de ces éléments l'était, puisque le référent des divinités exprimant les aspects de l'action était humain, ou que le Génie d'Auguste était vénéré à l'égal de celui du Peuple romain.

La règle répétée par les arvales ne méritait donc pas les sarcasmes de Sir Ronald. Conformément à l'enseignement de J. Rudhardt, elle est parfaitement logique et compréhensible, dès qu'elle est placée dans son contexte, dès qu'on "l'explique par la pensée antique elle-même ». Ce n'est pas son sens propre qu'il faut regarder, mais sa répétition même. Selon les conclusions de J.-M. Bertrand dans son étude sur les Lois de Platon ${ }^{26}$, une législation, même écrite, « n'avait pas de valeur en soi, puisque le groupe pouvait se sentir le droit de le corriger à son gré. Ce qui fondait la valeur d'une législation était en fait la réaffirmation permanente de ce qu'il fallait la maintenir».

John SCHEID

Collège de France

11, Place Marcelin-Berthelot

F - 75005 PARIS

Courriel:john.scheid@college-de-france.fr

${ }^{26}$ BERTRAND, o.c. (n. 16), p. 221-247, et notamment 246-247. 